\title{
Contribution to S-EMG Signal Compression in 1D by the Combination of the Modified Discrete Wavelet Packet Transform (MDWPT) and the Discrete Cosine Transform (DCT)
}

\author{
Colince Welba ${ }^{*}$, Aimé Joseph Oyobé Okassa², Pascal Ntsama Eloundou ${ }^{3}$, Pierre Ele ${ }^{4}$ \\ ${ }^{1}$ Department of Fundamental Science, Faculty of Mines and Petroleum Industries, University of Maroua, Maroua, Cameroon \\ ${ }^{2}$ School of Engineering of Masuku, Franceville, Gabon \\ ${ }^{3}$ Physics Department, Faculty of Sciences, University of Ngaoundere, Ngaoundere, Cameroon \\ ${ }^{4}$ Electrical Engineering and Telecommunications Department, National Advanced School of Engineering,University of Yaounde 1, \\ Yaoundé, Cameroon \\ Email:*welbacolince@yahoo.fr, oyobeokassa@yahoo.fr,pentsama@yahoo.fr,pierre_ele@yahoo.fr
}

How to cite this paper: Welba, C., Okassa, A.J.O., Eloundou, P.N. and Ele, P. (2020) Contribution to S-EMG Signal Compression in $1 \mathrm{D}$ by the Combination of the Modified Discrete Wavelet Packet Transform (MDWPT) and the Discrete Cosine Transform (DCT). Journal of Signal and Information Processing, 11, 35-57.

https://doi.org/10.4236/jsip.2020.113003

Received: June 28, 2020

Accepted: August 28, 2020

Published: August 31, 2020

Copyright $\odot 2020$ by author(s) and Scientific Research Publishing Inc. This work is licensed under the Creative Commons Attribution International License (CC BY 4.0).

http://creativecommons.org/licenses/by/4.0/

\begin{abstract}
A new Modified Discrete Wavelets Packets Transform (MDWPT) based method for the compression of Surface EMG signal (s-EMG) data is presented. A Modified Discrete Wavelets Packets Transform (MDWPT) is applied to the digitized s-EMG signal. A Discrete Cosine Transforms (DCT) is applied to the MDWPT coefficients (only on detail coefficients). The MDWPT+ DCT coefficients are quantized with a Uniform Scalar Dead-Zone Quantizer (USDZQ). An arithmetic coder is employed for the entropy coding of symbol streams. The proposed approach was tested on more than 35 actuals S-EMG signals divided into three categories. The proposed approach was evaluated by the following parameters: Compression Factor (CF), Signal to Noise Ratio (SNR), Percent Root mean square Difference (PRD), Mean Frequency Distortion (MFD) and the Mean Square Error (MSE). Simulation results show that the proposed coding algorithm outperforms some recently developed s-EMG compression algorithms.
\end{abstract}

\section{Keywords}

S-EMG, Compression, MDWPT, DCT, Arithmetic Coding, Uniform Scalar Dead-Zone Quantizer (USDZQ)

\section{Background}

Electromyographic signal compression is a recurrent topic in telemedicine. Digi- 
tized s-EMGs are most commonly used in applications such as monitoring and patient databases. Furthermore, long-term records are widely used to extract important information from the muscles signals or to detect such information. Therefore, the purpose of s-EMG signal compression is to reduce by as much as possible the number of bits of digitized s-EMG data that need to be transmitted or stored, with reasonable complexity of the implementation, while maintaining clinically acceptable signal quality. During the past few decades, many schemes for s-EMG compression have been proposed. As most of them do not reconstruct exactly the original signal when decoded, they are called lossy-compression techniques. Some authors [1] [2] have classified compression techniques of biomedical signals into two categories, namely direct methods and transform methods. However, some other publications, such as [3], have proposed a third category, "other methods", for those methods that cannot be included in the first two categories. We will not dwell on the details of the different categories here but we situate our compression approach in the second category, namely the transform methods. The state of the art of S-EMG signal compression, which we are going to present here, will be essentially based on transform methods. Waveform transformation-based encoders have shown better performance evaluation based on objective metrics versus Signal-to-Noise Ratio (SNR) and data compression ratio [4]. The Discrete Wavelet Transform (DWT) and the Discrete Cosine Transform (DCT) known for their energy concentration advantages, regularity in the transformed domain have been applied to S-EMG compression [5] [6] [7]. Wavelet transforms (associated with another transform) provide better results when applied to S-EMG encoders. The built-in EZW (Embedded Zero-tree Wavelets) compression algorithm is an example. It has a high compression ratio with low distortion of the EMG waveform [8] [9] [10]. Increasingly, studies are looking at optimizing basic functions to better decorrelate the S-EMG signal in the transform domain [11] [12] [13] [14]. Some researchers, in the interest of improving S-EMG compression techniques, have invested in a hybrid approach, thus modifying standard transform-based coders. For example, vector quantization has been applied to the transformed wavelet coefficient vector [15]. Another approach used mathematical models or neural networks [16] [17] [18] to approximate the shape of spectral amplitude in the wavelet domain. Another approach has modified the JPEG 2000 standard into 1D (one dimension) [6] to compress S-EMG. Using JPEG2000 [19] [20] [21] [6] [22]; H.264/AVC [12] [16]; HEVC [22]; 2D-DCT [7]; 2D-DWT [7]; and 2D-fractal [23] have also been reported in the literature. The standard JPEG compression algorithm approach for high density electromyography (HD EMG) was found in [24], and recurring patterns [25] for S-EMG 1D [26] and 2D S-EMG signals [22] were found in Literature. In addition, research involving compressed detection has also been found in the literature [27] [28] [29] [30]. The MDWPT (without the DCT) was applied for the first time for S-EMG signal compression in 2015 and was presented at an international conference (IUT-Entreprises 2015 from the University of Douala, Cameroon). It was clear from this work that the MDWPT is suitable for compress- 
ing the surface EMG signal with promising results. This first experiment resulted in a conference paper and was published in 2016 [31]. In the literature, we also find the work of Oyobe et al. [32]. Oyobe et al. compress surface EMG signals by combining the Wavelet Packet Transform (WPT) with the Discrete Cosine Transform (DCT). They did a comparative study between the combinations: WPT + DCT and DCT + WPT. It appears from their work that the best combination is the first one (WPT + DCT). This work is also appropriate as a reference for comparing the expected results under this Article. Although all this work has interesting solutions, the parameters of calculation complexity, compression factor, distortion rate and reconstruction fidelity still need to be improved to meet ideal specifications. It is therefore undoubtedly that the search for new techniques for s-EMG signal compression is still ongoing. The major contribution we make through this article is the improvement of PRD and acceptable data quality as well as the conservation of considerable information after reconstruction (PRD and visual observation). The efficiency of our approach will be evaluated with respect to the wavelet packet transform [32] and this evaluation will be extended with respect to the literature. The rest of the paper is organized as follows: Section 2 presents the method, Section 3 presents the results and discussion, and Section 4 contains the conclusion.

\section{Methods}

\subsection{Discrete Wavelet Packet Transform (DWPT)}

Discrete Wavelet Packet Transform (DWPT) (sometimes known as just wavelet packets) is a wavelet transform where the signal is passed through more filters than the Discrete Wavelet Transform (DWT). Wavelet packets are the particular linear combination of wavelets. They form bases which retain many of the orthogonality, smoothness, and localization properties of their parent wavelets. The coefficients in the linear combinations are computed by a recursive algorithm making each newly computed wavelet packet coefficient sequence the root of its own analysis tree.

In the DWT, each level is calculated by passing the previous approximation coefficients though a high and low pass filters. However, in the DWPT, both the detail and approximation coefficients are decomposed. Figure 1 shows decomposition into wavelet packets at level 3 .

The coefficients of detail $\left(d_{i}\right)$ are obtained by high-pass filtering (filter $h_{1}$ ), and decimated by 2 [33] [34]. The coefficientsof approximations $\left(a_{i}\right)$ are obtained in the same way by low-pass filtering (filter $g_{1}$ ).

The resolution at the output of each pair of filters is two times lower than the input resolution. This is the principle of dyadic multiresolution analysis. The Mallat S. algorithm for wavelet packets is a generalization of discrete wavelet decomposition that offers a rich range of possibilities for signal analysis [35]. For an $n$-level decomposition, there are $(n+1)$ possible ways to decompose or encode the signal [36]. The wavelet coefficients, the conjugate filters and the 


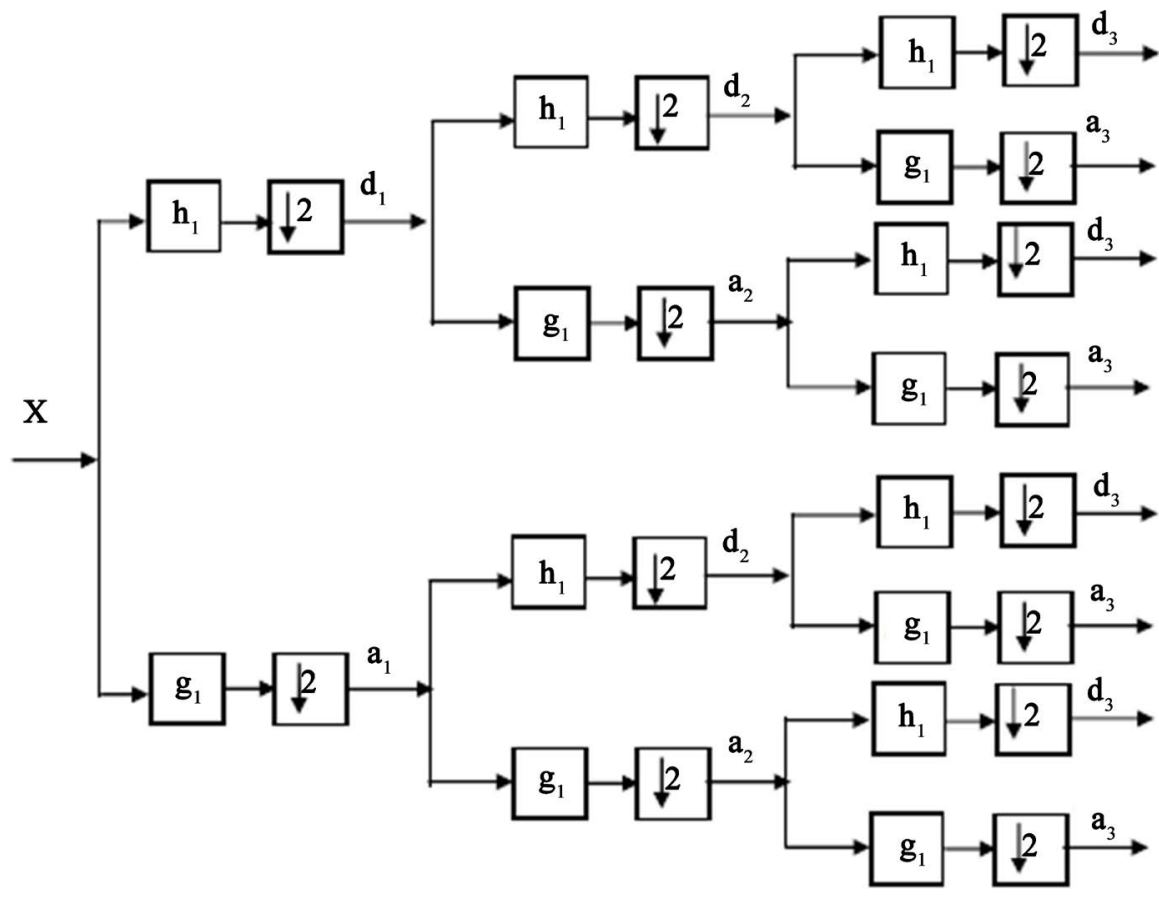

Figure 1. Wavelet Packet Decomposition Tree (level 3).

reconstruction of the signal are respectively determined by the Equations (1)-(5).

$$
\begin{aligned}
a[j-1, k] & =\sum_{n} h[n-2 k] a[j, n] \\
d[j-1, k] & =\sum_{n} g[n-2 k] a[j, n] \\
H[\omega] & =\sum_{k} h[k] \mathrm{e}^{-j k \omega} \\
G[\omega] & =\sum_{k} g[k] \mathrm{e}^{-j k \omega}
\end{aligned}
$$

with $G[\omega]$ the low pass filter, $H[\omega]$ the high pass filter, $a[j-1, k]$ the approximation coefficients, $d[j-1, k]$ the detail coefficients.

The reconstruction of the signal is given by the equation

$$
a[j, k]=\sum_{n} h[n-2 k] a[j-1, k]+\sum_{n} g[n-2 k] a[j-1, k]
$$

\subsection{Modified Discrete Wavelet Packet Transform (MDWPT)}

The algorithm of the MDWPT consists of modifying the pyramidal algorithm of the wavelet packet transform shown in Figure 1. Its principle is shown in Figure 2 .

Figure 2 takes as input an $\mathrm{X}$ signal. This input signal $(\mathrm{X})$ is decomposed by the DWT into detail coefficients (DC) and approximation coefficients (AC).

The approximation coefficients (AC) are then decomposed by a basic wavelet (lazy wavelet) into an even signal (even numbers) and an odd number (odd numbers 1). Lazy wavelet is a wavelet that separates a given signal into two sub-signals: a signal consisting of even index coefficients (even signal) and the 


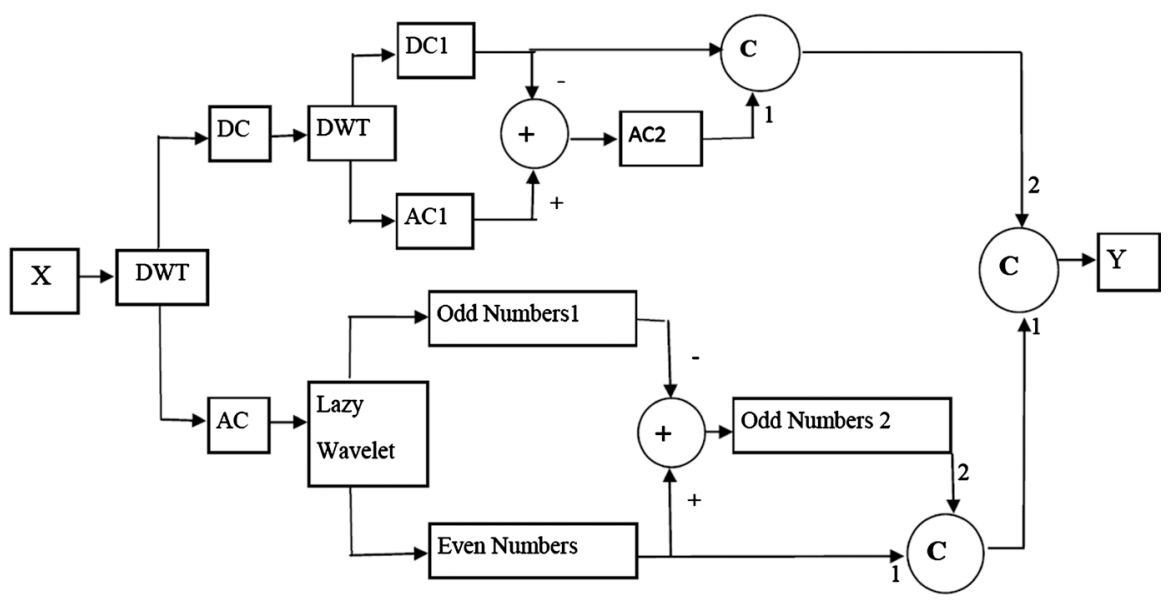

Figure 2. MDWPT.

other consisting of odd index coefficients (odd signal). This step can be considered as a subsampling of the input signal.

A subtraction is then made between the even signal and the odd signal (odd numbers 1 ) and the result is assigned to the odd signal 2 (odd numbers 2). The approximation coefficient (AC) is then reconstituted by concatenating the even number coefficients (even numbers) and the odd number (odd numbers 2 ) previously obtained.

About the detail coefficients (DC), they are decomposed by the wavelet transform, in detail coefficients (DC1) and in approximation coefficients (AC1). A subtraction is made between $\mathrm{DC} 1$ and $\mathrm{AC} 1$ and the result is assigned in $\mathrm{AC} 2$. Initials $\mathrm{DC}$ are reconstituted by concatenating $\mathrm{AC} 2$ and $\mathrm{DC} 1$.

C: Concatenation. The term concatenation designates the act of putting end to end at least two strings of characters.

The signal $Y$ is the concatenation of the new representation of DC and AC.

\subsection{Discrete Cosine Transform}

The discrete cosine transform decomposes the S-EMG signal into real coefficients in the frequency space. The direct and inverse transform of a signal $x(n)$ are carried out according to Equations (6) and (7) respectively and defined in [37].

$$
\begin{aligned}
& y(k)=\alpha(k) \sum_{n=0}^{N-1} x(n) \cos \left(\frac{(2 n+1) k \pi}{2 N}\right), k=0,1,2, \cdots, N-1 \\
& x(n)=\sum_{n=0}^{N-1} \alpha(k) y(k) \cos \left(\frac{(2 n+1) k \pi}{2 N}\right), n=0,1,2, \cdots, N-1
\end{aligned}
$$

the coefficient $\alpha$ takes the values according to Equation (8).

$$
\alpha(k)= \begin{cases}\frac{1}{\sqrt{N}} & \text { for } k=0 \\ \sqrt{\frac{2}{N}} & \text { for } k=1,2, \cdots, N-1\end{cases}
$$


The advantage of this transform is that it is real, reversible and has a fast calculation algorithm. DCT has excellent power concentration and bleaching of highly correlated data. Thus, it is widely used in compression of the S-EMG signal.

\subsection{Compression Approach}

The compression and decompression schemes are shown in Figure 3 and Figure 4 respectively. The method consists in decorrelating the original S-EMG signal by the MDWPT + DCT. The decorrelated signal is quantized with a uniform scalar dead-zone quantizer. An arithmetic coder is employed for the entropy coding of symbol streams.

Figure 3 shows the compression scheme that we propose.

The numbers 1 and 2 in front of the arrows, mean that during the concatenation, the vector bearing the number 1 is placed in the first position and is followed by the vector which is carried by the number 2 and so on;

The signal reconstruction algorithm is shown in Figure 4.

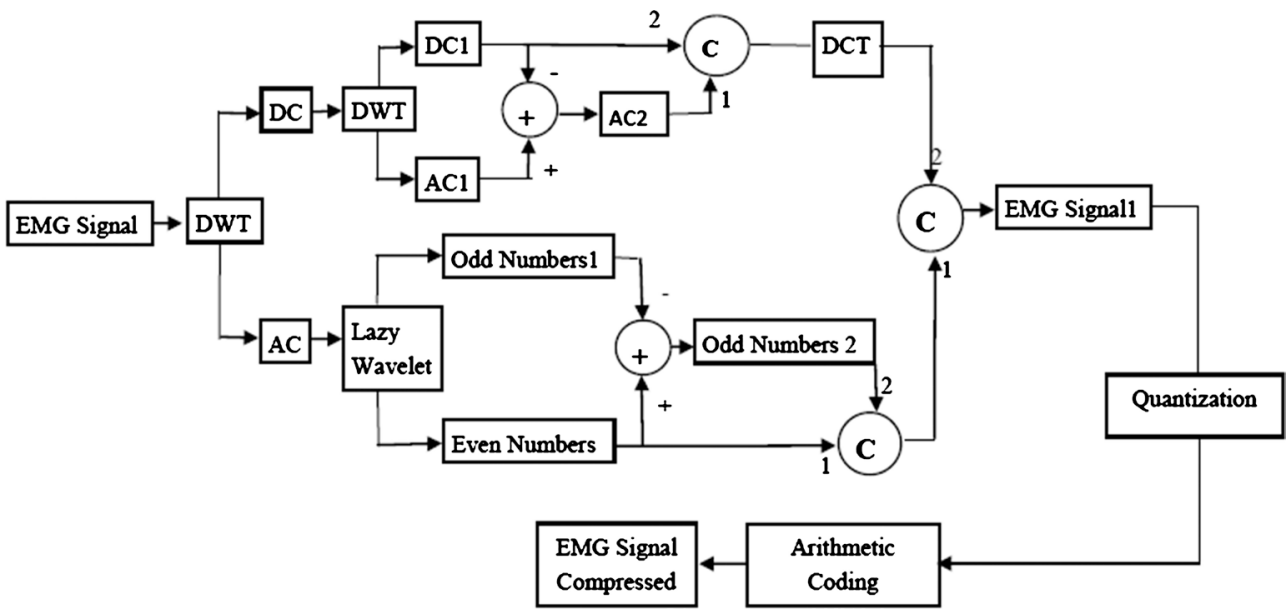

Figure 3. S-EMG signal compression diagram.

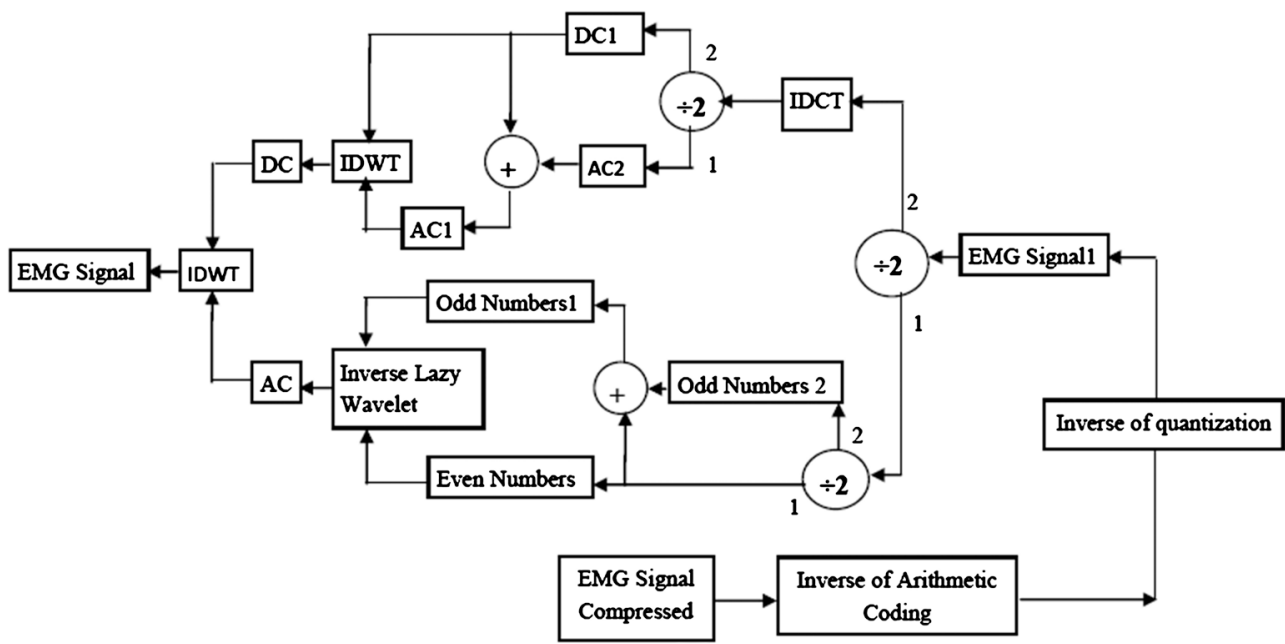

Figure 4. S-EMG decompression diagram. 
$\div 2$ : This symbol means that the input signal is divided into two signals of the same length.

\section{Choosing the position of the DCT}

During the experiment, we noticed that by applying the DCT on the approximation coefficients or on the detail coefficients. We applied our approach on the S-EMG signals of the different categories and the observation is almost the same.

Table 1 below presents some results (results obtained on the signal denominated Kher1 of category 1 ).

Table 1. Influence of the DCT on the coefficients of the MDWPT.

\begin{tabular}{ccccc}
\hline \multirow{2}{*}{$\begin{array}{c}\text { quantization } \\
\text { step }\left(\Delta_{b}\right)\end{array}$} & \multicolumn{2}{l}{ DCT on the coefficients of approximations } & \multicolumn{2}{l}{ DCT on the coefficients of details } \\
\cline { 2 - 5 } $10^{-4}$ & CF (\%) & PRD (\%) & CF (\%) & PRD (\%) \\
$10^{-5}$ & 97.02 & 27.7 & 97.39 & 26.82 \\
$10^{-6}$ & 92.76 & 0.29 & 93.72 & 2.75 \\
$10^{-7}$ & 87.56 & 0.03 & 88.24 & 0.28 \\
\hline
\end{tabular}

The results in terms of compression factor or PRD are presented.

Although the difference between the two results is not too great, it is important to note that the DCT on the detail coefficients is found to give slightly higher compression rates.

The implementation of the DCT on the detail coefficients would allow our algorithm to extract an excellent concentration of the little information that the detail coefficients contain. For this reason, we chose to implement the DCT only on the detail coefficients.

\subsection{Scalar Quantization with Dead Zone}

\section{Quantization}

The simplest form of quantization is scalar quantization. JPEG 2000 employs a dead-zone uniform scalar quantizer to coefficients resulting from the wavelet transform of image samples [38]. In this article, we employed dead-zone uniform scalar quantizer to coefficients resulting from the MDWPT + DCT of s-EMG samples namely EMG signal 1 . Figure 5 illustrates such a quantizer with step size $\Delta_{b}$ :

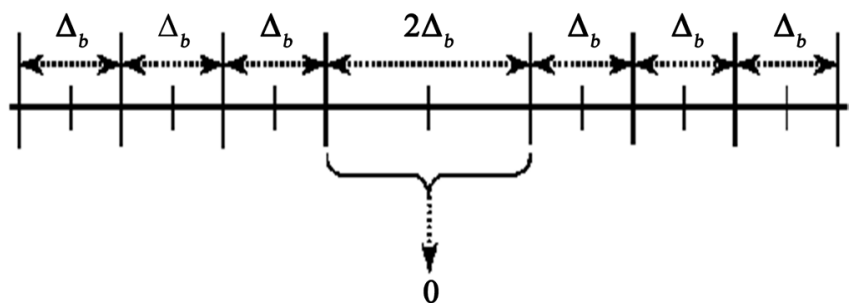

Figure 5. Definition of quantization zones and highlighting the dead zone. 
A scalar quantizer (SQ) can be described as a function $Q$ that maps each element in a subset of the real line to a particular value. For a given MDWPT + DCT coefficient EMG signal 1; the quantizer produces a signed integer $q$ given by

$$
q=Q(\text { EMG signal1) }
$$

The quantization index $q$ indicates the interval in which EMG signal 1 lies. In Figure 5, the endpoints of the quantization intervals are indicated by the vertical lines. Given $q$; the decoder produces an estimate of EMG signal 1 as

$$
\overline{\text { EMG signal1 }}=\overline{Q^{-1}}(q)
$$

In this work, EMG signal1 corresponds to the symbol flows from inverse arithmetic coding.

For a given step size $\Delta_{b} ; q$ is computed as

$$
q=Q(\text { EMG signal1 })=\operatorname{sign}(\text { EMG signal1 })\left[\frac{\mid \text { EMG signal1 } \mid}{\Delta_{b}}\right]
$$

The dimension of the quantization step $\Delta_{b}$ is represented with respect to the dynamic dimension of the sub-band b. $\Delta_{b}$ is represented in a form $\left(\varepsilon_{b}, \mu_{b}\right)$ [39] corresponding to:

$$
\Delta_{b}=\left(1+\frac{\mu_{b}}{2^{11}}\right) \cdot 2^{R_{b}-\varepsilon_{b}}
$$

$R_{b}$ is the dynamics of the original signal (number of bits), $\varepsilon_{b}$ is the desired dynamic of the coefficients and $\mu_{b}$ a multiplicative factor allowing to have values of $\Delta_{b}$ different from the multiples $2^{N}$, with $N$ positive integer.

Notice that the MDWPT+DCT coefficients inside the interval $\left(-\Delta_{b} ; \Delta_{b}\right)$ are quantized to zero for the quantizer in Figure 5. Thus, the interval $\left(-\Delta_{b} ; \Delta_{b}\right)$ is called the "deadzone". The width of this interval is $2 \Delta_{b}$; while all other intervals are of width $\Delta_{b}$. Figure 5 can be completed as follow Figure 6.

The inverse quantizer is given by

$$
\overline{\text { EMG signal1 }}=\overline{Q^{-1}}(q)= \begin{cases}0, & q=0, \\ \operatorname{sign}(q)(|q|+r) \Delta_{b}, & q \neq 0,\end{cases}
$$

where $r$ is a user selectable parameter within the range $0 \leq r<1$ (typically $r=1 / 2$ ). $r$ can be chosen to achieve the best objective or subjective quality at reconstruction.

- $r=0.5$ result in midpoint reconstruction (no polarization).

- $r<0.5$ polarization of the reconstruction towards zero.

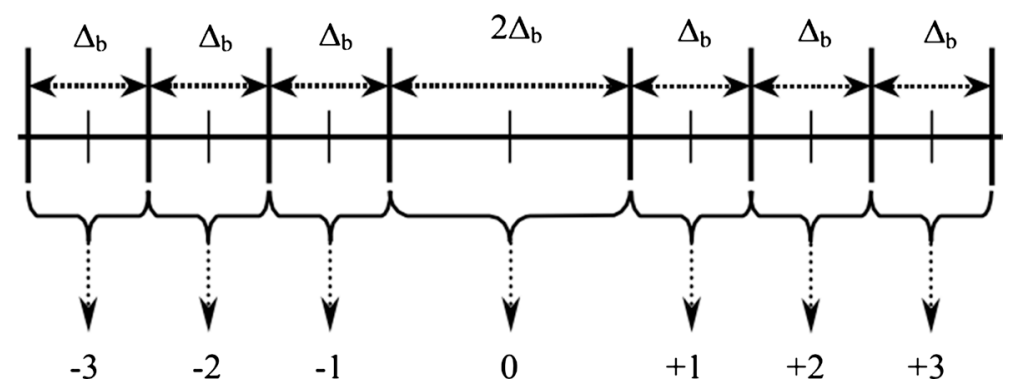

Figure 6. Quantification Indexes. 
A popular value for $r$ is 0.375 . for more details on the value of $r=0.375$, refer to $[38]$.

\subsection{Arithmetic Coding}

An arithmetic coder is employed for the entropy coding of symbol streams from Uniform Scalar Dead-Zone Quantizer (USDZQ). The Arithmetic coding allows, from the probability of occurrence of the symbols of a source to create a single code word that is associated with a sequence of arbitrary length symbols. This differs from the Huffman encoding that assigns code words to variable lengths to each source symbol. The associated code with a sequence is a real number in the interval $[0,1]$. This code is built by recursive subdivision of intervals. A range is divided for each new symbol belonging to the sequence. Is obtained, ultimately, a subinterval of the interval $[0,1]$ such that every real number belonging to this interval represents the sequence to coded.

\subsection{Evaluation Methods}

The Compression Factor (CF) is an important parameter in the quality evaluation of a compression algorithm. It is defined by:

$$
\mathrm{CF}=100 \% *\left(\frac{O_{S}-C_{S}}{O_{S}}\right)
$$

where $O_{S}$ is the number of bits needed to store the original data and $C_{S}$ the amount of bits needed to store the compressed data.

It is the main criterion for evaluating a compression algorithm. But when it comes to the evaluation of a lossy compression method, it is necessary to associate with this quantitative parameter those qualitative ones. Quality parameters are used to control the quality of reconstructed signals and to compare different approaches. The most commonly used quality measure is Mean Square Error (MSE) and defined by:

$$
\operatorname{MSE}=\frac{1}{N} \sum_{n=1}^{N}\left(y_{O}[n]-y_{r}[n]\right)^{2}
$$

$y_{O}[n]$ is the original signal (according Figure 3, $y_{O}[n]=$ EMG signal);

$y_{r}[n]$ is the reconstructed signal;

$N$ is the number of samples of the signal.

We have the signal to noise ratio (SNR):

$$
\mathrm{SNR}=10 \log _{10}\left(\frac{\sigma_{x}^{2}}{\sigma_{e}^{2}}\right)
$$

where log is decimal logarithm.

With $\sigma_{x}^{2}$ is the spectral power of the original signal and $\sigma_{e}^{2}$ is the spectral power of the reconstruction error.

In [5] [40] two other evaluation criteria of the quality of the reconstructed signal are presented: the distortion of the average frequency denoted MFD (Mean Frequency Distortion) and the PRD (Percent Root mean square Difference). 
They are defined respectively by Equations (17) and (18).

$$
\operatorname{MFD}=\left(\frac{\left|F_{o}-F_{r}\right|}{\max \left(F_{o}, F_{r}\right)}\right)^{2}
$$

In Formula (17), $F_{o}$ and $F_{r}$ represent the average frequency calculated respectively on the original signal and on the reconstructed signal.

$$
\operatorname{PRD}=\sqrt{\frac{\sum_{n=0}^{N-1}\left(y_{O}[n]-y_{r}[n]\right)^{2}}{\sum_{n=0}^{N-1}\left(y_{O}[n]-\mu\right)^{2}}} \cdot 100 \%
$$

$N$ is the number of samples of the original signal; $\mu$ is the reference value of the DAC (Digital Analog Converter) used for data acquisition $s(n)$ ( $\mu=0$ for EMG signals). $y_{r}[n]$ is reconstructed signal and $y_{O}[n]$ is the original signal.

\subsection{Characteristics and Sources of the Signals Used}

The compression algorithm proposed is applied to two categories of surface EMG signals. The first category contains surface EMG signals collected at a resolution of 12 bits/sample. The second category consists of EMG signals suitable for a dynamic and isometric protocol at resolution of 16 bits/sample. In the course of the experiments we found that the resolution of the signals influenced the different parameters such as PRD, CF... Therefore, we decided to apply our approach to both categories of EMG signals.

\subsubsection{S-EMG Protocol of Category 1}

The recordings are performed on biceps muscles with $40 \%$ of the maximum voluntary contraction. The angle between the arm and forearm of the subject is 90 $\therefore$. The electrical activity of the muscle via the electrodes passes through an amplifier whose gain is between 2000 and 5000 (enough to view the output voltage). The signal thus amplified, passes through an analog/digital converter and this signal is recovered by a computer and stored as a record. The signals are recorded at sampling rates of $2048 \mathrm{~Hz}$ and with a resolution of $12 \mathrm{bits} / \mathrm{sample}$.

\subsubsection{S-EMG of Category 2 Using Dynamic and Isometric Protocol}

The acquisition of the different EMG signals in this category respects dynamic and isometric experimental protocols.

\section{- Isometric experimental protocol}

The recording was carried out on 14 individuals. EMG signals are collected on the biceps muscles using pre-amplified surface electrodes (DE-02 model, DelSys Inc. Boston MA, USA). The angle between the arm and forearm of the subjects was $90^{\circ}$ with $60 \%$ of the maximum voluntary contraction. The signals were fed into a data acquisition card with LabVIEW (NI-DAQ for Windows, National Instruments, USA). All signals were sampled at $2 \mathrm{kHz}$ and digitized with 2 bytes/sample. The duration of the signals varies from 3 to 6 minutes [17].

\section{- Dynamic experimental protocol}

During the evaluation of the proposed techniques with a dynamic experimental 
protocol, a set of S-EMG signals collected on the large external muscle were used in 14 people on a cycling simulator (Cateye CS1000, USA). In the experiment, pre-amplified surface electrodes were used (DE-02 model, DelSys Inc. Boston, MA, USA). The signals were fed into a data acquisition card with LabVIEW (NI-DAQ for Windows, National Instruments, USA). All signals were sampled at $2 \mathrm{kHz}$ and quantized on 16 bits. The duration of the signals varies from 3 to 6 minutes [17].

\section{Results and Discussion}

\subsection{Results of Proposed Approach}

The results of compression and decompression are reported in Tables 2-4 and Figures 7-10.

The results that we present in the different tables below are some results selected from among many others. In each category of EMG signals, we have renamed the signals. in the tables below, the names "Kheir1", "Kher2", "Jouve3" refer to the signals of category 1. For category 2, the names "EMG Dynamic 1", "Dynamic 4", refer to names, dynamic S-EMGs and "EMG isometric 1", "EMG isometric 4" refer to names, isometric S-EMGs.

Tables 2-4 present respectively the results of the compression and decompression by the proposed approach (MDWPT + DCT) implemented on the S-EMG of the first category and the second category. The proposed method

Table 2. Results of the proposed approach implemented on S-EMG of the category 1 (12 bits/samples).

\begin{tabular}{|c|c|c|c|c|c|c|c|c|c|c|}
\hline \multirow{3}{*}{$\begin{array}{c}\text { Quantization } \\
\text { Step }\end{array}$} & \multicolumn{10}{|c|}{ Proposed method } \\
\hline & \multicolumn{5}{|c|}{ EMG Kheir 1} & \multicolumn{5}{|c|}{ EMG Kheir 2} \\
\hline & CF (\%) & PRD (\%) & SNR (dB) & MSE & MFD (\%) & CF (\%) & PRD (\%) & SNR (dB) & MSE & MFD (\%) \\
\hline $10^{-8}$ & 83.54 & 0.002 & 91.22 & $2.80 \mathrm{E}-17$ & $4.44 \mathrm{E}-9$ & 83.48 & 0.029 & 90.61 & $3.015 \mathrm{E}-17$ & $3.69 \mathrm{E}-8$ \\
\hline $10^{-7}$ & 87.03 & 0.03 & 70.97 & $2.96 \mathrm{E}-15$ & $3.54 \mathrm{E}-8$ & 86.92 & 0.028 & 70.88 & $2.83 \mathrm{E}-15$ & $1.67 \mathrm{E}-4$ \\
\hline $10^{-6}$ & 88.24 & 0.28 & 51.21 & $2.8 \mathrm{E}-13$ & $1.43 \mathrm{E}-5$ & 88.20 & 0.29 & 50.67 & $2.97 \mathrm{E}-13$ & 0.01 \\
\hline $10^{-5}$ & 93.72 & 2.75 & 31.22 & $2.79 \mathrm{E}-11$ & $2.40 \mathrm{E}-5$ & 93.63 & 2.85 & 30.89 & $2.83 \mathrm{E}-11$ & 0.02 \\
\hline $10^{-4}$ & 97.39 & 26.82 & 11.43 & $2.66 \mathrm{E}-9$ & $4.72 \mathrm{E}-4$ & 97.32 & 27.57 & 11.19 & $2.63 \mathrm{E}-9$ & 0.02 \\
\hline
\end{tabular}

Table 3. Results of the proposed algorithm implemented on S-EMG using Dynamic Protocol of the category 2.

\begin{tabular}{|c|c|c|c|c|c|c|c|c|c|c|}
\hline \multirow{2}{*}{$\begin{array}{c}\text { Quantization } \\
\text { Step }\end{array}$} & \multicolumn{10}{|c|}{ Proposed method } \\
\hline & \multicolumn{5}{|c|}{ EMG Dynamic 1} & \multicolumn{5}{|c|}{ EMG Dynamic 4} \\
\hline $10^{-5}$ & 88.34 & 0.0043 & 87.15 & $2.71 \mathrm{E}-11$ & $1.62 \mathrm{E}-8$ & 88.56 & 0.0047 & 86.42 & $3.11 \mathrm{E}-11$ & $3.82 \mathrm{E}-8$ \\
\hline $10^{-4}$ & 90.90 & 0.05 & 66.85 & $2.90 \mathrm{E}-9$ & $1.71 \mathrm{E}-8$ & 91.03 & 0.05 & 66.40 & $3.12 \mathrm{E}-9$ & $1.99 \mathrm{E}-5$ \\
\hline $10^{-3}$ & 92.79 & 0.43 & 47.26 & $2.58 \mathrm{E}-7$ & $2.16 \mathrm{E}-6$ & 93.36 & 0.43 & 47.42 & $2.46 \mathrm{E}-7$ & $8.26 \mathrm{E}-5$ \\
\hline $10^{-2}$ & 95.92 & 4.27 & 27.40 & $2.56 \mathrm{E}-5$ & $2.74 \mathrm{E}-5$ & 96.07 & 26.86 & 4.54 & $2.80 \mathrm{E}-5$ & 0.02 \\
\hline $10^{-1}$ & 98.42 & 37.42 & 8.54 & 0.0019 & 0.23 & 98.57 & 36.77 & 6.69 & 0.0018 & 3.22 \\
\hline
\end{tabular}


C. Welba et al.

Table 4. Results of the proposed algorithm implemented on S-EMG using Isometric protocol of the category 2.

\begin{tabular}{|c|c|c|c|c|c|c|c|c|c|c|}
\hline \multirow{3}{*}{$\begin{array}{c}\text { Quantization } \\
\text { Step }\end{array}$} & \multicolumn{10}{|c|}{ Proposed method } \\
\hline & \multicolumn{5}{|c|}{ EMG Isometric 1} & \multicolumn{5}{|c|}{ EMG Isometric 4} \\
\hline & CF (\%) & PRD (\%) & SNR (dB) & MSE & MFD (\%) & CF (\%) & PRD (\%) & SNR (dB) & MSE & MFD (\%) \\
\hline $10^{-2}$ & 88.67 & 0.006 & 83.82 & $3.05 \mathrm{E}-5$ & $8.62 \mathrm{E}-10$ & 88.91 & 0.01 & 81.98 & $2.99 \mathrm{E}-5$ & $1.05 \mathrm{E}-8$ \\
\hline $10^{-1}$ & 91.27 & 0.06 & 64.01 & 0.0029 & $2.89 \mathrm{E}-7$ & 91.51 & 0.08 & 62.01 & 0.0029 & $6.36 \mathrm{E}-8$ \\
\hline $2 * 10^{0}$ & 94.46 & 1.27 & 37.91 & 1.19 & $5.36 \mathrm{E}-5$ & 94.75 & 1.58 & 36.02 & 1.18 & $1.88 \mathrm{E}-7$ \\
\hline $4^{*} 10^{0}$ & 95.35 & 2.47 & 32.14 & 4.50 & $1.73 \mathrm{E}-4$ & 95.62 & 3.15 & 30.03 & 4.70 & 0.0038 \\
\hline $6^{*} 10^{0}$ & 95.85 & 3.87 & 28.24 & 11.05 & $3.9 \mathrm{E}-4$ & 96.10 & 4.79 & 26.39 & 10.87 & $7.62 \mathrm{E}-4$ \\
\hline
\end{tabular}
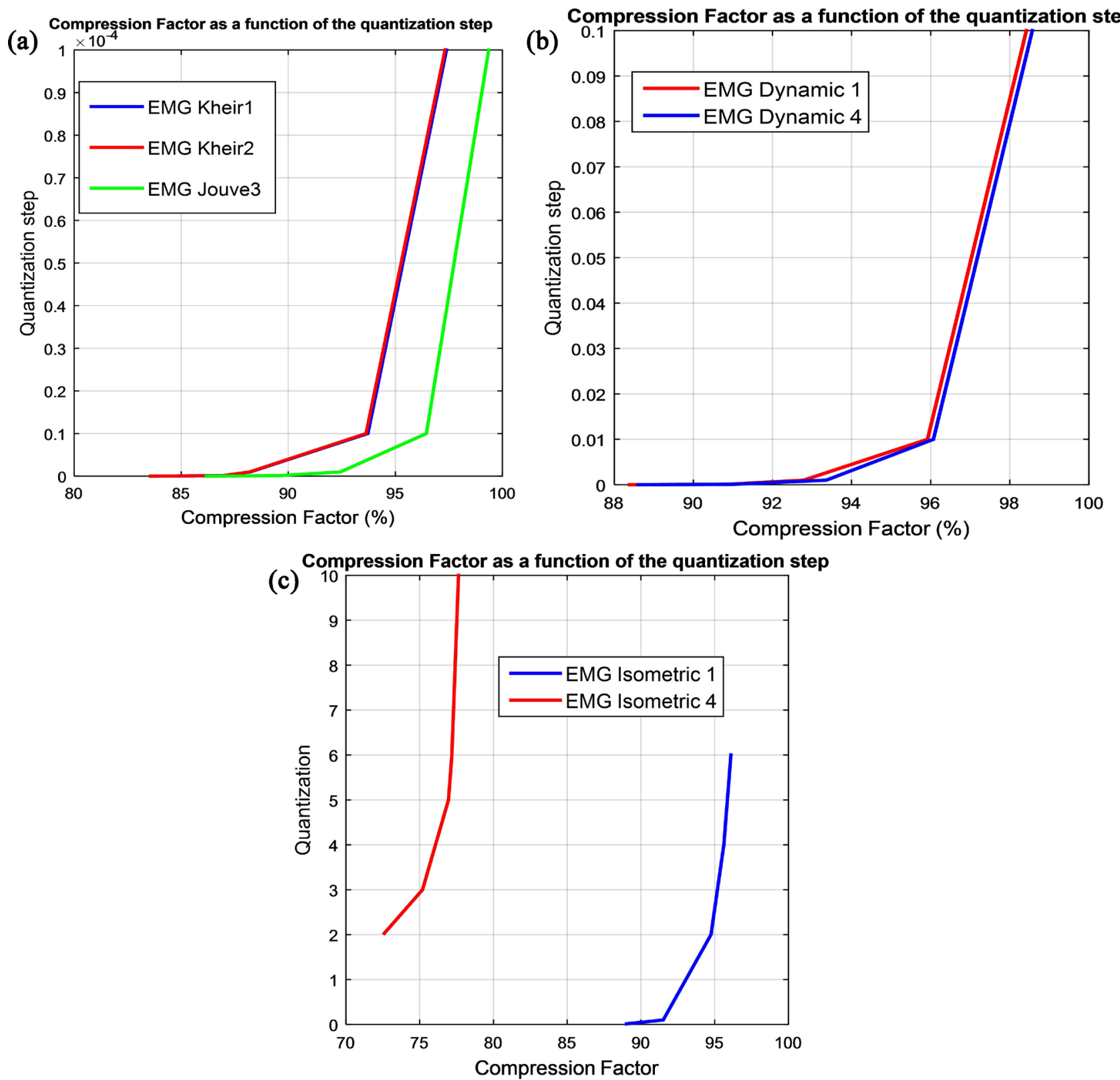

Figure 7. Evolution of the CF versus the quantization step. (a) S-EMG of the first category; (b) Dynamic S-EMG of the second category; (c) Isometrics S-EMG of the second category. 
gives good results, be it qualitative (MSE, SNR, PRD and MFD) or quantitative (CF). According to these Tables 2-4, the quality of the signal improves progressively with the refinement of the quantization step and this to the detriment of the compression factor which decreases as the quantization step is refined. The compression factor will be chosen according to the application. Thus, it is necessary to make a compromise between the compression factor and the quality of the decompressed signal to be retained. It should not be forgotten that the quality of the signal reconstructed by this algorithm depends closely on the refinement of the quantization step. The computational load depends on the refinement of the quantization step. Finally, to choose the compression factor it is imperative to consider the application to choose the quality of the signal and the associated compression rate. The framed parts of Tables 2-4 above, represent the results where the quality of the reconstructed signal is good. The evolution of the compression factor as a function of the quantization step for each category of the EMG signals is represented by the following figures.

Figures 7(a)-(c) above shows the evolution of the compression factor and the quantization step for each category of the signal. The analysis of Figures 7(a)-(c) and Tables 2-4 shows that a small step corresponds to a good quality of the reconstructed signal and therefore an optimal compression factor. An excessive quantization step corresponds to a very high compression factor and consequently a poor quality of the reconstructed signal. This leads us to look for the optimal quantization step corresponding to the best quality of the reconstructed signal and indirectly the optimal compression factor. During the experiment, we determined that the optimal compression factor is on average $93.42 \%$. With regard to the qualitative parameters, the averages are: $\mathrm{PRD}=1.06 \%$; $\mathrm{SNR}=40.63 \mathrm{~dB}$. This at first sight shows that the proposed approach is efficient and robust. However, it is important to note that the quality of the decompressed signal is very sensitive when it is a compression for transmission or for storage for the purpose of remote diagnostics or subsequent diagnoses. It follows from the foregoing that the trade-off between the compressed signal and the quality of the decompressed signal must be closely monitored, since poor quality of the reconstructed signal would lead to a fatal diagnosis error.

It is therefore imperative that subjective criteria come into play requiring the presence of experts accustomed to evaluate these criteria. Thus, in the biomedical field where the final judge is the specialist, the subjective criterion should be based on an expertise and diagnosis of original signals and reconstructed signals after compression. To better understand the quality of the signal reconstructed by the subjective criterion (visual aspect), the results of this experiment are recorded in Figures 8-10, representing the plot of the two categories of signals used.

The goal of lossy compression is to drastically reduce the size of the data while keeping the compressed signal as close to the original as possible to better analyze the resemblance between the reconstructed compressed signal and the original signal, we use the visual aspect. so we superimposed the two signals. From this 

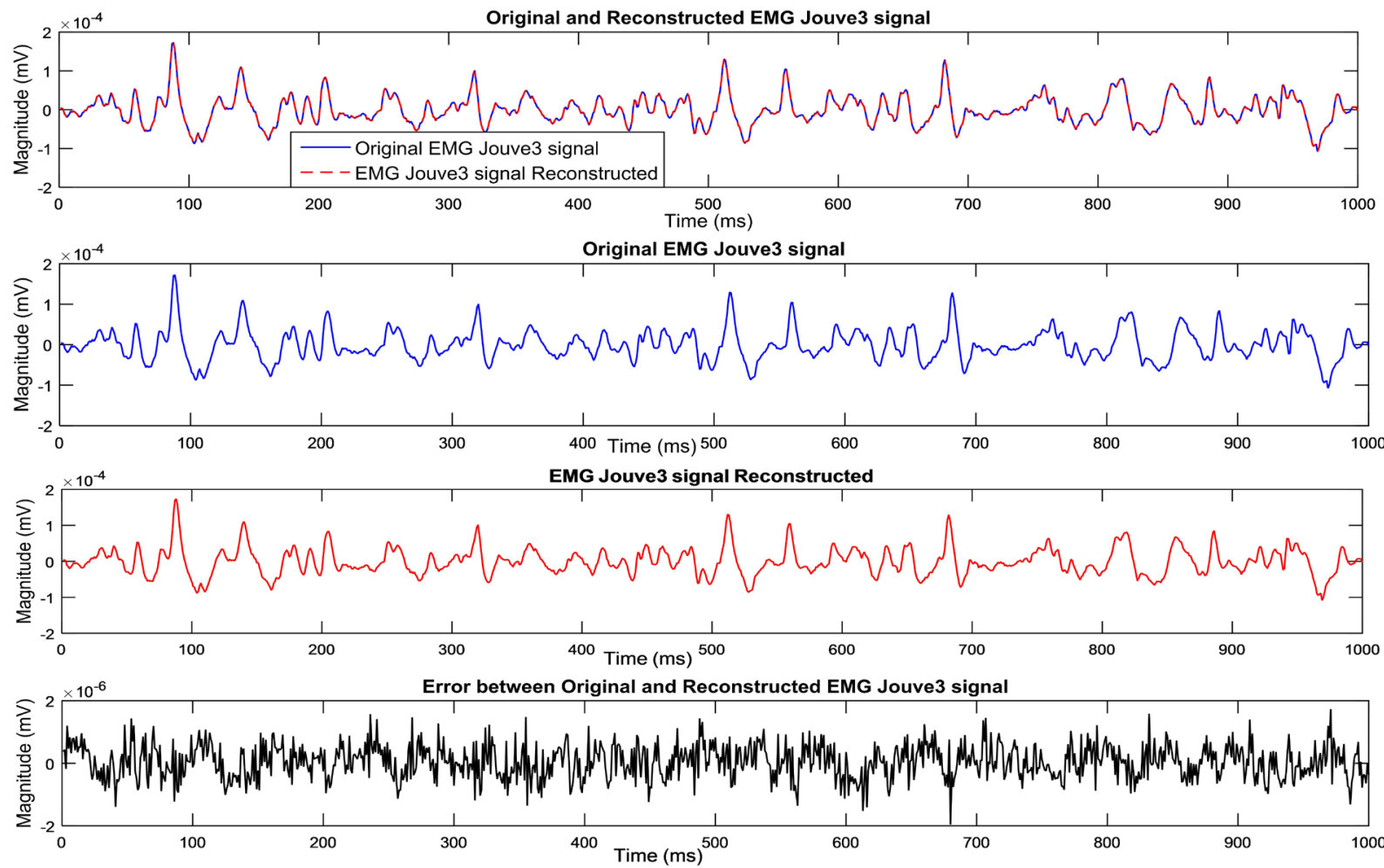

Figure 8. Qualitative examples. EMG signal (Jouve3) of the first category. $\mathrm{CF}=92.44 \%, \mathrm{PRD}=1.48 \%, \mathrm{SNR}=36.57 \mathrm{~dB}, \mathrm{MSE}=$ $3.15 \times 10^{-13}, \mathrm{MFD}=0.01 \%$, Quantization step $=10^{-6}$.
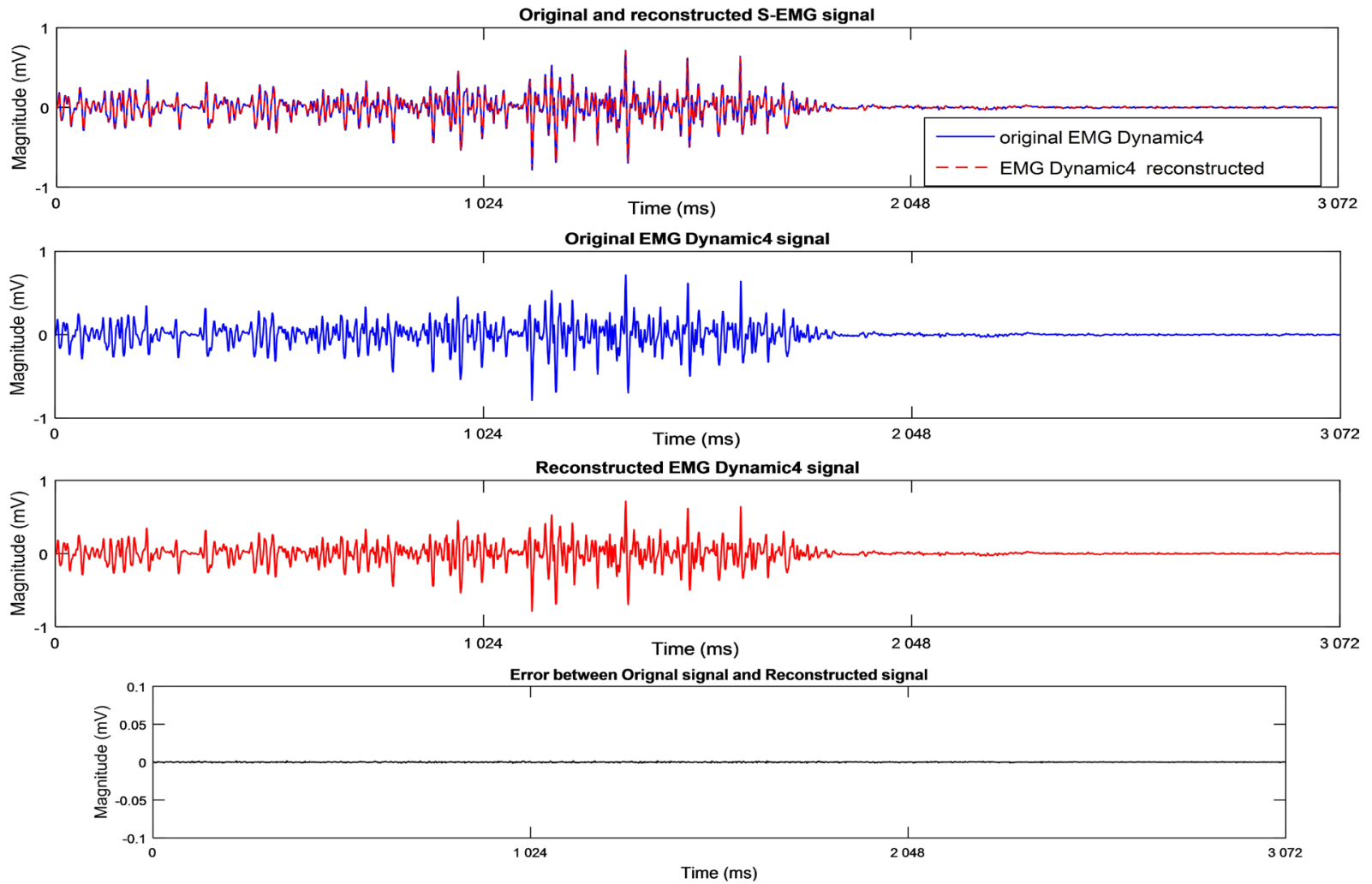

Figure 9. Qualitative examples. EMG signal (Dynamic4) of the third category. $\mathrm{CF}=93.36 \%, \mathrm{PRD}=0.43 \%, \mathrm{SNR}=47.42 \mathrm{~dB}, \mathrm{MSE}$ $=2.46 \times 10^{-7}, \mathrm{MFD}=8.26 \times 10^{-5}$, Quantization step $=10^{-3}$. 

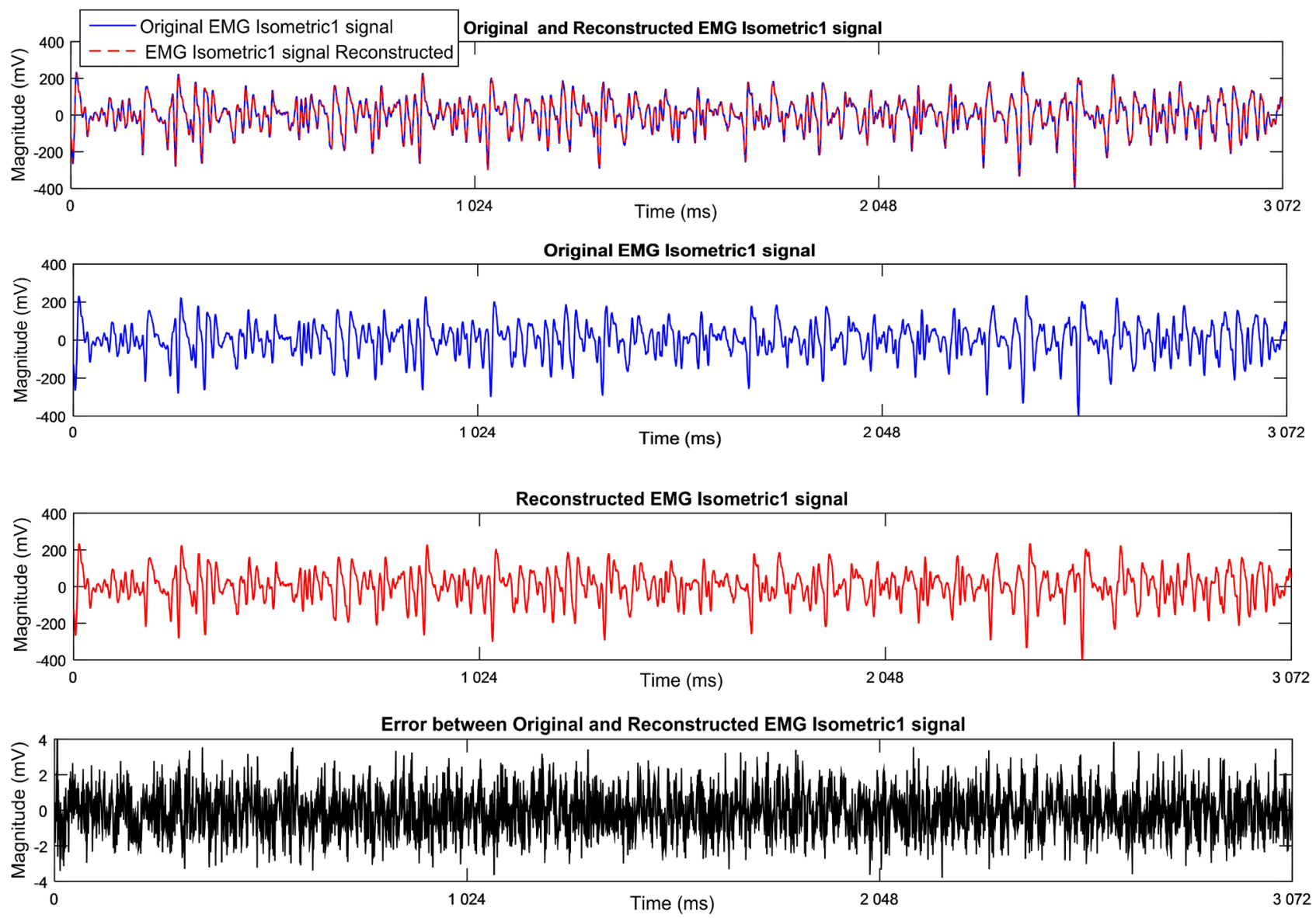

Figure 10. Qualitative examples. EMG signal (Isometric1) of the second category. $\mathrm{CF}=94.46 \%, \mathrm{PRD}=1.27 \%, \mathrm{SNR}=37.91 \mathrm{~dB}$, MSE $=1.19, \mathrm{MFD}=5.36 \times 10^{-5}$, Quantization step $=2$.

superposition, we find that the two signals are almost identical. If we take the visual aspect as a criterion, we can say that Figures 8-10 above show that the proposed approach for different signals guarantees the conservation of considerable information after reconstruction (CF, PRD and visual observation). Although the results presented below show that the proposed approach is effective quantitatively and qualitatively in compressing surface EMG signals, it is imperative to compare these performances with the scientific works reported in the literature.

\subsection{Comparative Performance of Proposed Approach with Others Works of Literature}

In order to make a comparative evaluation of the performance of the proposed approach with other published works, we will distinguish three (03) cases. The first case will be a comparison of the performance of the proposed approach with the work published on the S-EMG signals of category 1 . The second case compares the proposed approach with literature's works implemented on the S-EMG signals of Category 2 (isometric protocol S-EMG signals), and the third and final case will be a comparison of the proposed approach with literature's work implemented on the S-EMG signals of Category 2 (dynamic protocol S-EMG signal). 


\section{- First comparison case (on S-EMG signals of category 1)}

The works [23] [31] and [32] mentioned in Table 5 and graphically represented in Figure 11 have all been implemented on the same S-EMG signals as the proposed approach. They are based on fractals, Modified Wavelet Packets Transforms (MDWPT) and WPT + DCT (Wavelet Packets Transforms + Discrete Cosine Transforms) respectively.

In analyzing the results, we note that the work of Ntsama et al. [23] have a minimum PRD of $1.09 \%$ corresponding to a CF of $70.80 \%$; Welba et al. [31] has a minimum $\mathrm{PRD}$ of $0.61 \%$ and $\mathrm{CF}=70 \%$; Oyobe et al. [32] has a minimum PRD of $2.44 \%$ for $\mathrm{CF}=72.52 \%$ while the proposed approach gives a minimum $\mathrm{PRD}$ of $0.002 \%$ for a CF of $83.54 \%$. It is obvious that the proposed approach is more

Table 5. Comparative performance evaluation for signals of the first Category-CF (\%) and PRD (\%). Best results (lowest PRD and high CF).

\begin{tabular}{cccccccc}
\hline \multicolumn{2}{c}{$\begin{array}{c}\text { Oyobeet al. [32] } \\
\text { WPT + DCT }\end{array}$} & \multicolumn{2}{c}{$\begin{array}{c}\text { Welba [31] } \\
\text { MDWPT }\end{array}$} & \multicolumn{2}{c}{$\begin{array}{c}\text { Ntsama [23] } \\
\text { FRACTAL }\end{array}$} & \multicolumn{2}{c}{$\begin{array}{c}\text { Proposed } \\
\text { MDWPT + DCT }\end{array}$} \\
\hline CF (\%) & PRD (\%) & CF (\%) & PRD (\%) & CF (\%) & PRD (\%) & CF (\%) & PRD (\%) \\
\hline 72.52 & 2.44 & 70 & 0.61 & 70.80 & 1.09 & 83.54 & 0.002 \\
75.19 & 2.45 & 75 & 1.19 & 71.82 & 4.01 & 87.03 & 0.03 \\
76.95 & 2.45 & 80 & 1.52 & 72.87 & 4.92 & 88.24 & 0.28 \\
77.18 & 2.45 & 85 & 2.68 & 73.73 & 6.65 & 93.72 & 2.75 \\
77.63 & 2.45 & $/$ & $/$ & 74.30 & 6.60 & 97.30 & 26.82 \\
\hline
\end{tabular}

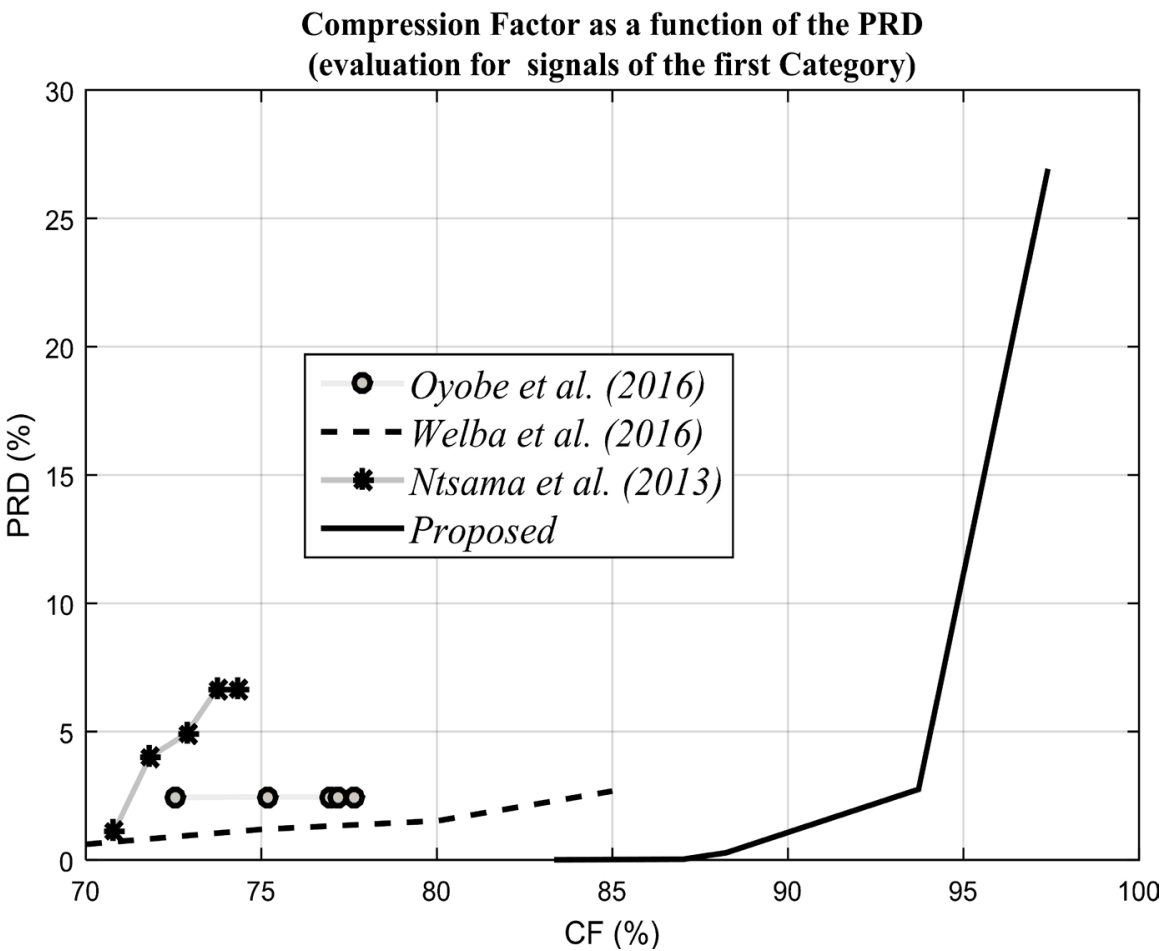

Figure 11. Compression Factor as a function of the PRD (evaluation for S-EMG signals of the first category). 
efficient than the published works [23] [31] and [32]. This comparison highlights the role, choice and importance of the DCT in the proposed approach on the one hand and the effectiveness of the approach compared to conventional wavelet packets on the other hand (Table 5 and Figure 11).

\section{- Second comparison case (on S-EMG signals with isometric protocol)}

Articles [16] [22] and [30] have been implemented on the same data bank but not necessarily on the same S-EMG signals. Using the same S-EMG data bank is one of the reasons that encouraged the comparison of the proposed approach with these works. A comparative analysis shows that the proposed approach seems more efficient than the works [16] [22] and [30] (Table 6 and Figure 12).

Table 6. Comparative performance evaluation for isometric protocol-PRD (\%). Best results (lowest PRD and high CF).

\begin{tabular}{|c|c|c|c|c|c|c|c|c|c|}
\hline \multicolumn{2}{|c|}{$\begin{array}{c}\text { Trabuco et al. } \\
\text { [22] }\end{array}$} & \multicolumn{2}{|c|}{ Melo et al. [30] } & \multicolumn{2}{|c|}{$\begin{array}{c}\text { Trabuco et al. } \\
\text { 1D WDAL }\end{array}$} & \multicolumn{2}{|c|}{$\begin{array}{l}\text { Trabuco et al. }[16] \\
\text { 2D RI-HEVC }\end{array}$} & \multicolumn{2}{|c|}{$\begin{array}{c}\text { Proposed } \\
\text { MDWPT + DCT }\end{array}$} \\
\hline $\mathrm{CF}$ & PRD & $\mathrm{CF}$ & PRD & $\mathrm{CF}$ & PRD & $\mathrm{CF}$ & PRD & $\mathrm{CF}$ & PRD \\
\hline 70 & 2.07 & 70 & - & 70 & 0.77 & 70 & - & 88.91 & 0.01 \\
\hline 75 & 2.22 & 75 & 1.65 & 75 & 1.24 & 75 & 1.21 & 91.51 & 0.08 \\
\hline 80 & 2.52 & 80 & 2.23 & 80 & 1.99 & 80 & 1.78 & 94.75 & 1.58 \\
\hline 85 & 3.31 & 85 & 3.38 & 85 & 3.36 & 85 & 2.99 & 95.62 & 3.15 \\
\hline 90 & 6.88 & 90 & 6.14 & 90 & 7.06 & 90 & 6.18 & 96.10 & 4.79 \\
\hline 95 & 19.74 & 95 & - & 95 & 19.28 & 95 & 18.3 & - & - \\
\hline
\end{tabular}

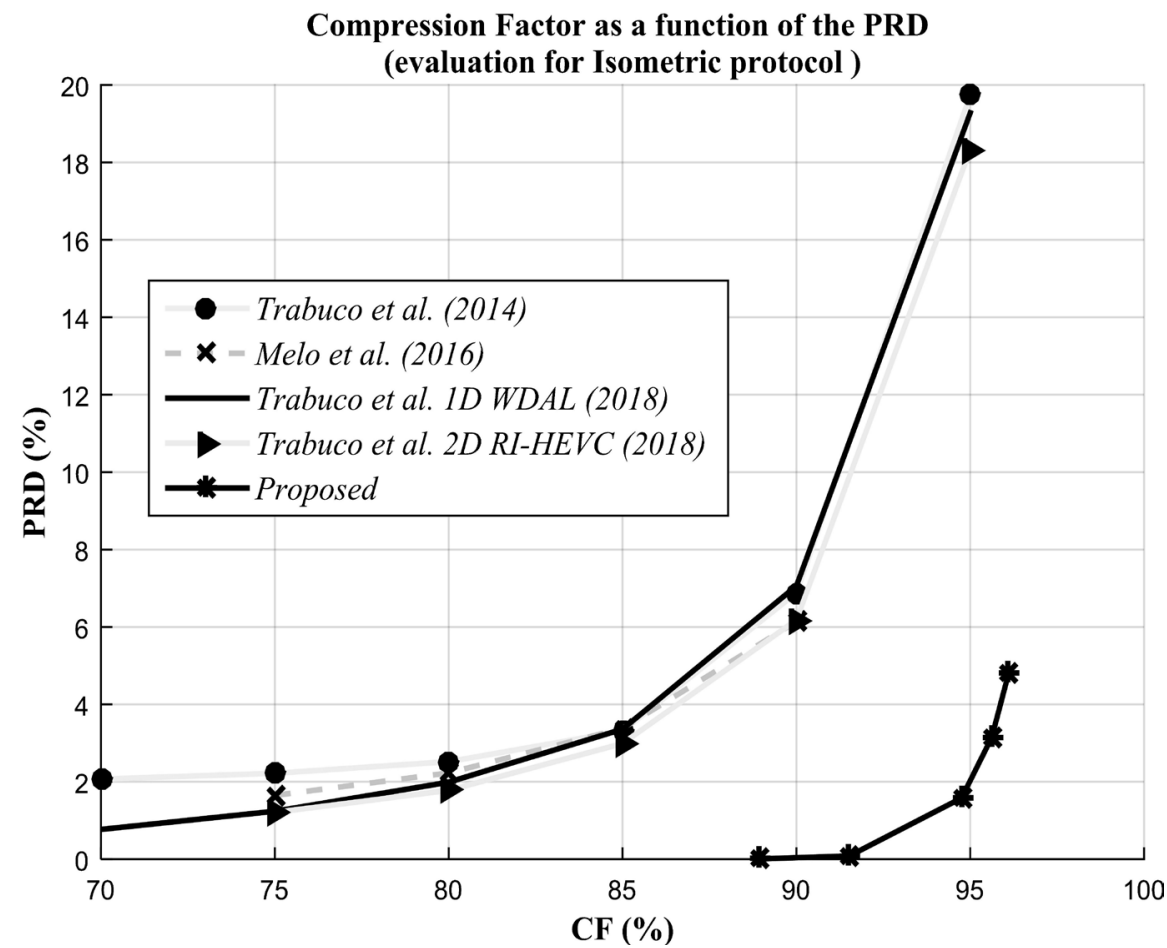

Figure 12. Compression Factor as a function of the PRD (evaluation for isometric protocol S-EMG signals of the second category). 
- Third comparison case (on S-EMG signals with dynmic protocol of the second category of S-EMG signals

For a given CF or less than $90 \%$, Figure 13 shows that the proposed approach has the lowest PRD among the different approaches in Table 7. This remark leads to the conclusion that the proposed approach is more efficient than the other approaches in Table 7 in terms of PRD. Despite the good performance, it is also important to note that our compression approach has certain limitations. For example, the average optimal compression ratio is $93.42 \%$ for an average PRD of $1.06 \%$ and an SNR of $40.63 \mathrm{~dB}$. From a compression ratio of $95 \%$, the quality of the reconstructed signal begins to deteriorate.

Table 7. Comparative performance evaluation for dynamic protocol-PRD (\%). Best results (lowest PRD and high CF).

\begin{tabular}{cccccccccc}
\hline \multicolumn{2}{c}{$\begin{array}{c}\text { Trabuco } \\
\text { et al. [36] }\end{array}$} & \multicolumn{2}{c}{$\begin{array}{c}\text { Melo } \\
\text { et al. [30] }\end{array}$} & \multicolumn{2}{c}{ Trabuco et al. [16] } & \multicolumn{2}{c}{ Trabuco et al. [16] } & \multicolumn{2}{c}{ Proposed } \\
2D WDL & 2D R-HEVC & MDWPT + DCT \\
\hline CF & PRD & CF & PRD & CF & PRD & CF & PRD & CF & PRD \\
\hline 70 & 4.41 & 70 & - & 70 & 1.12 & 70 & - & 88.56 & 0.0047 \\
75 & 4.70 & 75 & 4.71 & 75 & 1.74 & 75 & - & 91.03 & 0.05 \\
80 & 5.41 & 80 & 6.25 & 80 & 2.64 & 80 & 2.71 & 93.36 & 0.43 \\
85 & 6.40 & 85 & 8.91 & 85 & 3.93 & 85 & 4.28 & 96.07 & 26.86 \\
90 & 8.22 & 90 & 12.60 & 90 & 6.11 & 90 & 7.96 & 98.57 & 36.77 \\
95 & 15.76 & 95 & - & 95 & 12.63 & 95 & 19.53 & - & - \\
\hline
\end{tabular}

Compression Factor as a function of the PRD (evaluation for Dynamic protocol )

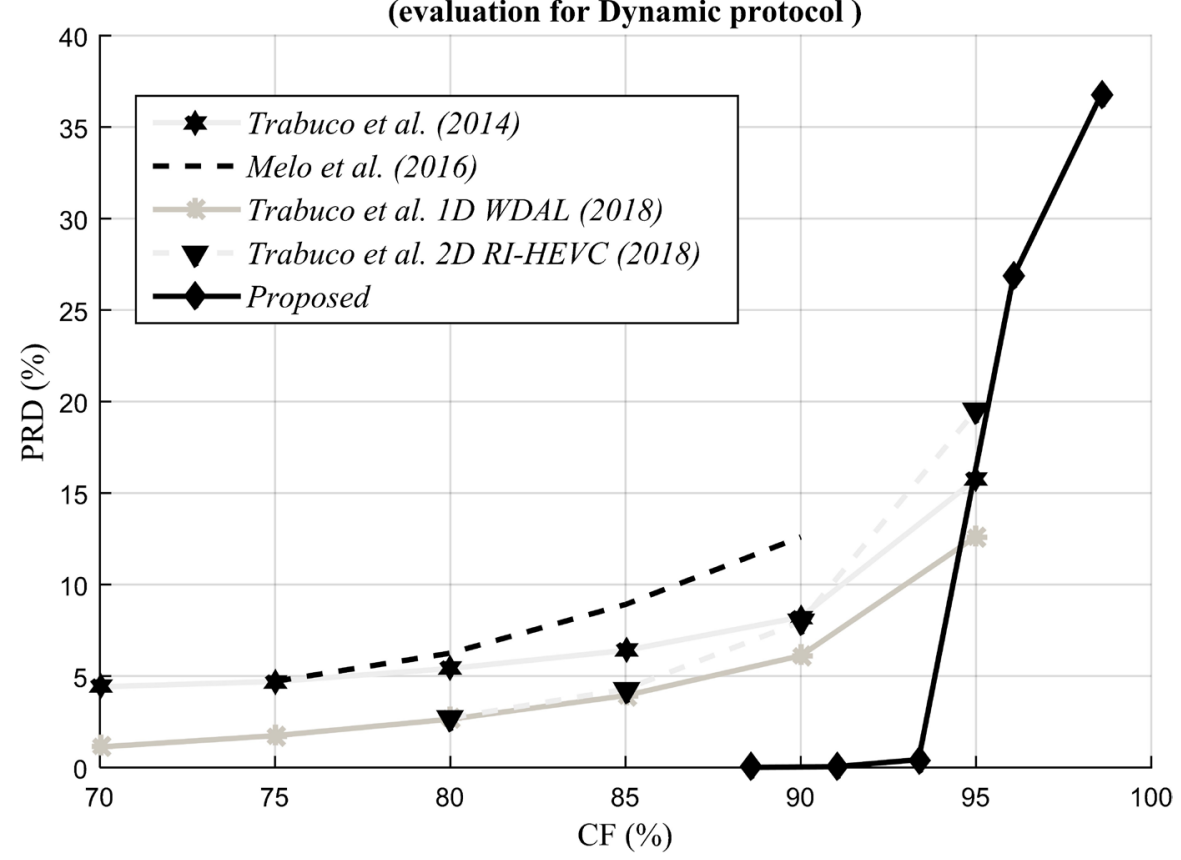

Figure 13. Compression Factor as a function of the PRD (evaluation for dynamic protocol S-EMG signals of the second category). 


\section{Conclusions}

In this article, it was a question of contributing to the compression of S-EMG signals through a new compression technique called MDWPT. This technique was tested on S-EMG signals in 2016 through a communication [31] which aimed to show that it is possible to compress S-EMG signals by this new approach (MDWPT). The purpose of this article was to further improve this compression technique on the one hand and compare its efficiency compared to conventional wavelet packets and compared to some literature work on the other hand. This work shows that the results are satisfactory and very encouraging. It is also apparent from this article that, the proposed algorithm for different signal guarantees acceptable quality as well as the conservation of considerable information after reconstruction (FC, PRD and visual observation). The performance of an S-EMG compression algorithm depends of the type and resolution of the S-EMG signal.

In this work, only surface EMG signals were considered. Extension to other types of electrophysiological signals may be a generalization track of the algorithm.

\section{Acknowledgements}

The authors are grateful to thank the volunteers of their participation. The authors wish to thank Professor Francisco Assis De Oliveira Nascimento for his collaboration and for providing the isometric and dynamic EMG signals used in this work.

\section{Conflicts of Interest}

The authors declare no conflicts of interest regarding the publication of this paper.

\section{References}

[1] Lu, Z., Kim, D.Y. and Pearlman, W.A. (2000) Wavelet Compression of ECG Signals by the Set Partitioning in Hierarchicaltreesalgorithm. IEEE Transactions on Biomedical Engineering, 47, 849-856. https://doi.org/10.1109/10.846678

[2] Nygaard, R., Melnikov, G. and Katsaggelos, A.K. (2001) A Rate Distortion Optimal ECG Coding Algorithm. IEEE Transactions on Biomedical Engineering, 48, 28-40. https://doi.org/10.1109/10.900246

[3] Blanco-Velasco, M., Cruz-Roldán, F., López-Ferreras, F., Bravo-Santos, A. and Martínez-Muñoz, D. (2004) A Low Computational Complexity Algorithm for ECG Signal Compression. Medical Engineering \& Physics, 26, 553-568. https://doi.org/10.1016/j.medengphy.2004.04.004

[4] Guerrero, A.P. and Mailhes, C. (1997) On the Choice of an Electromyogram Data Compression Method. Proceedings of the 19th Annual International Conference of the IEEE Engineering in Medicine and Biology Society, Chicago, 30 October-2 November 1997, 1558-1561. https://doi.org/10.1109/IEMBS.1997.757009

[5] Berger, P.A, Nascimento, F.A.O., Carmo, J.C., Rocha, A.F. and dos Santos, I. (2003) Algorithm for Compression of EMG Signals. Proceedings of the 25th Annual Inter- 
national Conference of the IEEE Engineering in Medicine Biology Society, 17-21 September 2003, Cancun, Mexico, 1299-1302. https://doi.org/10.1109/IEMBS.2003.1279508

[6] Ntsama, E.P., Ele, P., Dieudonné, S.Z. and Tonye, E. (2013) Evaluation of EMG Signals Compression by JPEG 2000 Called 1D. International Journal of Engineering and Technology, 5, 44-51.

[7] Ntsama, E.P., Ele, P., and Kabiena, I.B.V. (2013) Compression Approach of EMG Signal Using 2D Discrete Wavelet and Cosine Transforms. American Journal of Signal Processing, 3, 10-16.

[8] Wellig, P., Cheng, Z., Semling, M. and Moschytz, G.S. (1998) Electromyogram Data Compression Using Single-Tree and Modified Zero-Tree Wavelet Encoding. Proceedings of the 20th Annual International Conference of the IEEE Engineering in Medicine and Biology Society, Hong Kong, China, 1 November 1998, 1303-1306. https://doi.org/10.1109/IEMBS.1998.747117

[9] Chan, A. and Englehart, K.B. (2005) Continuous Myoelectric Control for Powered Protheses Using Hidden Markov Models. IEEE Transactions on Biomedical Engineer, 52, 121-124. https://doi.org/10.1109/TBME.2004.836492

[10] Norris, J.A., Englehart, K.B. and Lovely, D.F. (2003) Myoelectric Signal Compression Using Zero-Trees of Wavelet Coefficients. Medical Engineering \& Physics, 25, 739-746. https://doi.org/10.1016/S1350-4533(03)00118-8

[11] Brechet, L., Lucas, M.F., Doncarli, C. and Farina, D. (2007) Compression of Biomedical Signals with Mother Wavelet Optimization and Best-Basis Wavelet Packet Selection. IEEE Transactions on Biomedical Engineering, 54, 2186-2192. https://doi.org/10.1109/TBME.2007.896596

[12] Paiva, J.P.L.M., Kelencz, C.A., Paiva, H.M., Galvão, R.K.H. and Magini, M. (2008) Adaptive Wavelet EMG Compression Based on Local Optimization of Filter Banks. Physiological Measurement, 29, 843-856. https://doi.org/10.1088/0967-3334/29/7/012

[13] Oyobé-Okassa, A.J. and Elé, P. (2014) Optimization of the Compression Ratio of the Modified Algorithm of Decomposition Electromyographic Signals by a Superimposed Coding. Proceedings of the 2014 Tenth International Conference on Signal-Image Technology and Internet-Based Systems, Marrakech, Morocco, 23-27 November 2014, 83-93. https://doi.org/10.1109/SITIS.2014.107

[14] Sarkar, S. and Bhoi, A.K. (2012) Compression of Surface Electromyographic Signal Using Wavelet Packet 1D. International Journal of Pharmaceutical Sciences and Health Care, 4, 115-121.

[15] Jain, N. and Vig, R. (2007) Wavelet Based Vector Quantization with Tree Code Vectors for EMG Signal Compression. Proceedings of the 6 th WSEAS International Conference on Signal Process, Dallas, TX, 22-24 March 2007, 117-124.

[16] Trabuco, M.H., Costa, M.V.C., Macchiavello, B. and de Oliveira Nascimento, F.A. (2018) S-EMG Signal Compression in One-Dimensional and Two-Dimensional Approaches. IEEE Journal of Biomedical and Health Informatics, 22, 1103-1113. https://doi.org/10.1109/JBHI.2017.2765922

[17] Trabuco, M.H., Costa, M.V.C., Nascimento, F.A. and de Oliveira Nascimento, F.A. S-EMG Signal Compression Based on Domain Transformation and Spectral Shape Dynamic Bit Allocation. BioMedical Engineering OnLine, 13, Article No. 22. https://doi.org/10.1186/1475-925X-13-22

[18] Berger, P.A., de Oliveira Nascimento, F.A., Rocha, A.F. and Carvalho, J.L.A. (2007) A New Wavelet-Based Algorithm for Compression of EMG Signals. Proceedings of 
the 2007 29th Annual International Conference of the IEEE Engineering in Medicine and Biology Society, Lyon, 22-26 August 2007, 1554-1557.

https://doi.org/10.1109/IEMBS.2007.4352600

[19] Costa, M.V.C., Berger, P.A., Rocha, A.F., de Carvalho, J.L.A. and de Oliveira Nascimento, F.A. (2008) Compression of Electromyographic Signals Using Image Compression Techniques. Proceedings of the 200830 th Annual International Conference of the IEEE Engineering in Medicine and Biology Society, Vancouver, BC, 20-25 August 2008 , 2948-2951. https://doi.org/10.1109/IEMBS.2008.4649821

[20] Costa, M.V.C., Carvalho, J.L.A., Berger, P.A., Zaghetto, A., Roch, A.F. and Nascimento, F.A.O. (2009) Two-Dimensional Compression of Surface Electromyographic Signals Using Column-Correlation Sorting and Image Encoders. Proceedings of the 2009Annual International Conference of the IEEE Engineering in Medicine and Biology Society, Minneapolis, MN, 3-6 September 2009, 428-431. https://doi.org/10.1109/IEMBS.2009.5333134

[21] Melo, W.C., Filho, E.B.L. and Júnior, W.S.S. (2012) Electromyographic Signal Compression Based on Preprocessing Techniques. Proceedings of the 2012 Annual International Conference of the IEEE Engineering in Medicine and Biology Society, San Diego, CA, 28 August-1 September 2012 , 5404-5407. https://doi.org/10.1109/EMBC.2012.6347216

[22] Melo, W.C., Filho, E.B.L. and Júnior, W.S.S. (2016) SEMG Signal Compression Based on Two Dimensional Techniques. BioMedical Engineering OnLine, 15, Article No. 41. https://doi.org/10.1186/s12938-016-0158-1

[23] Ntsama, E.P., Lionel, T.M., Ele, P. and Kabiena, I.B. (2013) EMG Signal Compression Using 2D Fractal. International Journal of Advanced Technology and Engineering Research, 3, 58-68.

[24] Itiki, C., Furuie, S.S. and Merletti, R. (2014) Compression of High-Density EMG Signals for Trapezius and Gastrocnemius Muscles. BioMedical Engineering OnLine, 13, Article No. 25. https://doi.org/10.1186/1475-925X-13-25

[25] de Carvalho, M.B., da Silva, E.A.B. and Finamore, W.A. (2002) Multidimensional Signal Compression Using Multiscale Recurrent Patterns. Signal Process, 82, 1559-1580. https://doi.org/10.1016/S0165-1684(02)00302-X

[26] Filho, E.B.L., Silva, E.A.B. and Carvalho, M.B. (2008) On EMG Signal Compression with Recurrent Patterns. IEEE Transactions on Biomedical Engineering, 55, 1920-1923. https://doi.org/10.1109/TBME.2008.919729

[27] Salman, A., Allstot, E.G., Chen, A.Y., Dixon, A.M.R., Gangopadhyay, D. and Allstot D.J. (2011) Compressive Sampling of EMG Bio-Signals. Proceedings of the 2011 IEEE International Symposium of Circuits and Systems, Rio de Janeiro, 15-18 May 2011, 2095-2098. https://doi.org/10.1109/ISCAS.2011.5938011

[28] Allstot, E.G., Chen, A.Y., Dixon, A.M.R., Gangopadhyay, D., Mitsuda, H. and Allstot, D.J. (2011) Compressed Sensing of ECG Bio-Signals Using One-Bit Measurement Matrices. Proceedings of the 2011 IEEE 9 th International New Circuits and systems conference, Bordeaux, 26-29 June 2011, 213-216.

https://doi.org/10.1109/NEWCAS.2011.5981293

[29] Dixon, A.M.R., Allstot E.G., Gangopadhyay, D. and Allstot D.J. (2012) Compressed Sensing System Considerations for ECG and EMG Wireless Biosensors. IEEE Transactions on Biomedical Circuits and Systems, 6, 156-166. https://doi.org/10.1109/TBCAS.2012.2193668

[30] Chen, Y.-S., Lin, H.-Y., Chiu, H.-C. and Ma, H.-P. (2014) A Compressive Sensing Framework for Electromyogram and Electroencephalogram. 2014 IEEE International 
Symposium on Medical Measurements and Applications, Lisboa, Portugal, 11-12 June 2014, 1-6. https://doi.org/10.1109/MeMeA.2014.6860096

[31] Welba, C., Ntsama, E.P. and Ele, P. (2016) Compression des Signaux Electromyographiques par la Transformée en Paquets d'ondelettes Modifiée. Sciences, Technologies et Développement, Edition Spéciale, 17, 194-198. http://www.univ-douala.com/

[32] Oyobe-Okassa, A.J., Abessolo, D.A. and Elé, P. (2016) Compression of EMG Signals by Superimposing Methods: Case of WPT and DCT. International Journal of Engineering and Technology, 8, 2319-8613.

[33] Ahmed, N. and Rao, K.R. (1975) Orthogonal Transforms for Digital Signal Processing. Springer, Berlin, Heidelberg. https://doi.org/10.1007/978-3-642-45450-9

[34] Cohen, A., Daubechies, I., Jawerth, B. and Vial, P. (1993) Multiresolution Analysis, Wavelets and Fast Algorithms on an Interval. Comptes Rendus de P Académie des Sciences, Série I, 316, 417-421.

[35] Goswami, J.C. and Chan, A.K. (1999) Fundamentals of Wavelets: Theory, Algorithms, and Applications. In: Wiley Series in Microwave and Optical Engineering, Wiley Interscience, Hoboken, NJ.

[36] Mallat, S.G. (1989) A Theory for Multiresolution Signal Decomposition: The Wavelet Decomposition. IEEE Transactions on Pattern Analysis and Machine Intelligence, 11, 674-693. https://doi.org/10.1109/34.192463

[37] de Melo, W.C., de Lima Filho, E.B. and da Silva Júnior, W.S. (2016) Electromyographic Signal Compression through Image Encoders and Preprocessing Techniques. Journal of Communication and Information Systems, 31, 188-197. https://doi.org/10.14209/jcis.2016.17

[38] Marcellin, W.M., Lepley, M.A., et al. (2002) An Overview of Quantization in JPEG 2000. Signal Processing. Image Communication, 17, 3-48. https://doi.org/10.1016/S0923-5965(01)00024-8

[39] Medouakh, S. and Baarir, Z.-E. (2007) Compression d'images fixes par la norme jpeg2000. JIG2007-3èmes Journées Internationales sur P Informatique Graphique, LESIA Laboratoire de Recherche, Département Electronique, Université de Biskra, Alegria. 10-15.

[40] Carotti, E.S.G., De Martin, J.C., Farina, D. and Merletti, R. (2005) Linear Predictive Coding of Myoelectric Signals. IEEE International Conference on Acoustics, Speech, and Signal Processing, Philadelphia, PA, 23-23 March 2005, 629-632. https://doi.org/10.1109/ICASSP.2005.1416382 


\section{Abbreviations}

$\begin{array}{ll}\text { MDWPT } & \text { Modified Discrete Wavelet Packet Transform } \\ \text { DCT } & \text { Discrete Cosine Transform } \\ \text { S-EMG } & \text { Surface electromyographics signal } \\ \text { CF } & \text { Compression Factor } \\ \text { SNR } & \text { Signal to noise Ratio } \\ \text { PRD } & \text { ADPCM: Adaptive Differential Pulse Code Modulation } \\ \text { ACELP } & \text { Algebraic Code-Excited Linear Prediction } \\ \text { MFD } & \text { Mean Frequency Distortion } \\ \text { PRD } & \text { Percent Root mean square Difference } \\ \text { MSE } & \text { Mean Square Error } \\ \text { DC } & \text { Detail Coefficients } \\ \text { AC } & \text { Approximation Coefficients } \\ \text { DAC } & \text { Digital Analog Converter } \\ \text { JPEG } & \text { Joint Photographic Experts Group } \\ \text { HD } & \text { High Density } \\ \text { HEVC } & \text { High Efficient Video Coding } \\ \text { H.264/AVC } & \text { Advanced Video Coding }\end{array}$

